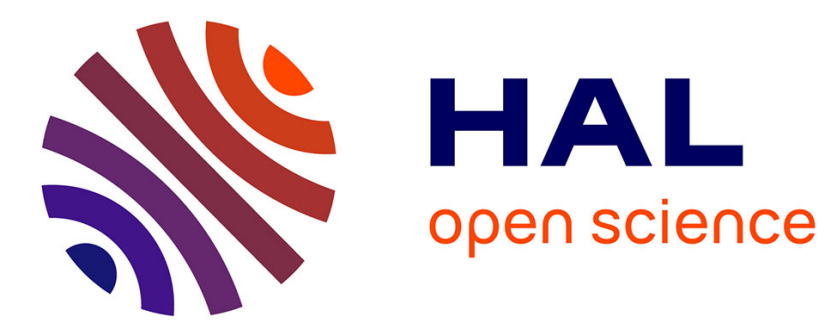

\title{
Criteria on the artificial compliance inherent to the intrinsic cohesive zone
}

\author{
Nawfal Blal, Loïc Daridon, Yann Monerie, Stéphane Pagano
}

\section{To cite this version:}

Nawfal Blal, Loïc Daridon, Yann Monerie, Stéphane Pagano. Criteria on the artificial compliance inherent to the intrinsic cohesive zone. Comptes Rendus Mécanique, 2011, 339 (12), pp.789-795. 10.1016/j.crme.2011.10.001 . hal-00672818

\section{HAL Id: hal-00672818 https://hal.science/hal-00672818}

Submitted on 22 Feb 2012

HAL is a multi-disciplinary open access archive for the deposit and dissemination of scientific research documents, whether they are published or not. The documents may come from teaching and research institutions in France or abroad, or from public or private research centers.
L'archive ouverte pluridisciplinaire HAL, est destinée au dépôt et à la diffusion de documents scientifiques de niveau recherche, publiés ou non, émanant des établissements d'enseignement et de recherche français ou étrangers, des laboratoires publics ou privés. 


\title{
Criteria on the artificial compliance inherent to the intrinsic cohesive zone
}

\author{
N. BLAL ${ }^{a, b, c}$, L. DARIDON ${ }^{a, c}$, Y. MONERIE ${ }^{b, c}$, S. PAGANO PA $^{a, c}$ \\ $a$ Laboratoire de Mécanique et Génie Civil, Université Montpellier 2, CNRS, CC 048 Place Eugène Bataillon, \\ 34095 Montpellier cedex \\ $b$ Institut de Radioprotection et de Sûreté Nucléaire, Bat. 702, CE Cadarache, BP3-13115 St. Paul-lez-Durance \\ Cedex \\ $c$ Laboratoire de Micromécanique et d'Intégrité des Structures, MIST Laboratory, IRSN-CNRS-Université \\ Montpellier 2 \\ \{nawfal.blal,loic.daridon,stephane.pagano\}@univ-montp2.fr, yann.monerie@irsn.fr
}

\begin{abstract}
This Note presents criteria on the artificial compliance due to intrinsic cohesive zone models. A homogenized model is proposed for a collection of cohesive zones embedded between each mesh of a finite element-type discretization (cohesive-volumetric approach). The overall elastic behavior of this cohesive-volumetric medium is obtained as a function of the local properties and the mesh size. For an isotropic discretization, a criterion on the cohesive stiffnesses is derived: the additional compliance inherent to intrinsic cohesive zone models is bounded by lower value.
\end{abstract}

keywords: damage; Cohesive Zone Model; Rigorous Criteria; Cohesive Stiffness; Homogenization

\section{Résumé}

Critères sur les raideurs cohésives pour les formulations intrinsèques. Cette Note propose des critères pour la souplesse additionnelle inhérente aux modèles de zones cohésives intrinsèques. Un modèle micromécanique est développé pour une collection de zones cohésives insérées entre toutes les mailles d'une discrétisation spatiale de type éléments finis (approche cohésive-volumique). Le comportement linéaire effectif du milieu cohésif-volumique est relié aux propriétés locales et à la taille de maille. Pour une discrétisation isotrope, un critère est obtenu pour les raideurs cohésives: la souplesse additionnelle macroscopique liée à la présence de modèles cohésifs est minorée.

Mots-clés: Endommagement; Modèle de Zone Cohésive; Critères rigoureux; Raideur cohésive; Homogénéisation

\section{Version française abrégée}

Les modèles de zone cohésive intrinsèque possèdent une raideur initiale finie: leur insertion entre chaque maille d'une discrétisation de type éléments finis (l'approche cohésive-volumique) conduit à l'introduction d'une souplesse additionnelle. Différents auteurs ont proposé de limiter cette souplesse additionnelle en définissant des critères reliant la raideur cohésive à la taille des maillages considérés, e.g. (2; 13); ces critères sont jusqu'à présent semi-empiriques et limités à certains types de chargement. Cette Note présente des critères valables pour tout maillage et tout taux de triaxialité.

Modèle micromécanique. Le principe de l'approche, proposé en premier lieu par (1), consiste à considérer l'approche cohésive-volumique comme une collection d'inclusions cohésives distribuée dans un milieu continu selon une statistique de distribution spatiale liée à la morphologie du maillage sous-jacent. Le comportement élastique homogène équivalent de ce milieu est borné par valeur inférieure à l'aide de l'estimation de Hashin-Shtrikman (4). Ce changement d'échelles nécessite de définir une déformation de la phase cohésive au travers d'une épaisseur fictive $e$ destinée à tendre vers zéro et un tenseur de 
raideur du quatrième ordre pour la phase cohésive (5). Une prise de moyenne adaptée de l'estimation de Hashin-Shtrikman sur les orientations des arêtes du maillage et un passage à la limite $e \rightarrow 0$ permet d'obtenir les critères attendus (12).

Critères. Dans le cas d'une microstructure globalement isotrope - phase continue isotrope et orientations équiprobables arêtes des mailles - un critère (16) est obtenu pour la raideur cohésive:

$$
\frac{C_{\mathrm{N}} L_{\mathrm{mesh}}}{E^{M}} \geq \gamma \frac{R}{1-R}\left(1+\frac{4}{3} \frac{C_{\mathrm{N}}}{C_{\mathrm{T}}}\right)
$$

où $C_{\mathrm{N}}$ et $C_{\mathrm{T}}$ sont les raideurs cohésives normale et tangente, $E^{M}$ le module de Young de la phase continue, $R$ une perte de raideur apparente tolérée par l'utilisateur, $L_{\text {mesh }}$ la taille de maille et $\gamma$ un paramètre lié à la morphologie du maillage. Les critères semi-empiriques de la littérature $« C_{\mathrm{N}} L_{\mathrm{mesh}} / E^{M}$ plus grand qu'une quantité fixée $\gg(2 ; 13)$ sont ainsi étendus et trouvent une justification théorique.

\section{Introduction}

Cohesive zone models (CZM) are one of the most widely used approaches to simulate numerical fracture processes from crack initiation to overall failure. In particular, the cohesive-volumetric finite element method allows multi-crack initiation without any ad hoc criteria: a traction-separation law is incorporated between each mesh of a finite element-type discretization. However, the numerical results show that this cohesive-volumetric implementation exhibits a strong mesh sensitivity which is still an issue of concern. The paper (12) was the first one to emphasize this mesh-dependency by illustrating the link between the crack path and the mesh size or the mesh type. Despite this crack path sensitivity, the authors show therein that global characteristics are not very sensitive to the mesh and can be predicted with reasonable accuracy.

This mesh-dependency is particularly noteworthy for intrinsic CZM, i.e. traction-separation laws with initial slope: embedding such types of CZM within a finite element mesh leads to an additional compliance that drastically increases when the mesh size decreases. Following (10), this artificial compliance can be illustrated on a simple 1D-example (see Fig. 1). In this case, the equilibrium condition reads: $F / S=E^{M} \varepsilon=C_{\mathrm{N}}[\boldsymbol{u}]$, where $F$ is the applied force, $\varepsilon$ the strain in the bulk elements (mesh size $L_{\text {mesh }}$, cross area $S$ ), $E^{M}$ the Young modulus of the bulk elements, $C_{\mathrm{N}}$ the initial cohesive stiffness of the intrinsic model and $[\boldsymbol{u}]$ is the displacement jump across the cohesive zone. From the definition of the overall strain

$$
\widetilde{\varepsilon}=\frac{F / S}{E^{M}}+\frac{F / S}{C_{\mathrm{N}} L_{\text {mesh }}},
$$

one can deduce the normalized apparent Young modulus:

$$
\frac{\widetilde{E}}{E^{M}}=\frac{F / S}{E^{M} \varepsilon}=\frac{\xi}{1+\xi} \quad \text { with } \quad \xi=\frac{C_{\mathrm{N}} L_{\mathrm{mesh}}}{E^{M}} .
$$

According to (1), the ratio $\xi$ seems to be the key adimensional parameter controlling the mesh sensitivity in intrinsic CZMs: more precisely, the added compliance vanishes $\left(\widetilde{E} \rightarrow E^{M}\right)$ when $\xi \rightarrow+\infty$, see Fig. 1 right. In other words, the overall elastic behavior is not affected by the introduction of intrinsic CZMs between bulk elements and therefore the mesh sensitivity effect vanishes.

Following the same set of ideas, various authors have proposed semi-empirical bounds for the ratio $\xi$ in order to obtain 'invisible' CZMs on a structural scale. Performing numerical tension and shear tests, (2) have noticed that the elastic wave speeds are unchanged across a cohesive line between two elastic and isotropic media when $\xi \geq 10$. Estimating the added compliance for cross-triangle elements arranged in quadrilateral pattern submitted to uniaxial tension, biaxial uniform tension and pure shear, (13) obtain ( $\nu^{M}$ is the Poisson ratio of the bulk material):

$$
\xi \gg \alpha \frac{\sqrt{2}+1}{1-\nu^{M}} \quad \text { with } \alpha=1 \text { for plane stress and } \alpha=\frac{1}{1-\left(\nu^{M}\right)^{2}} \text { for plane strain. }
$$



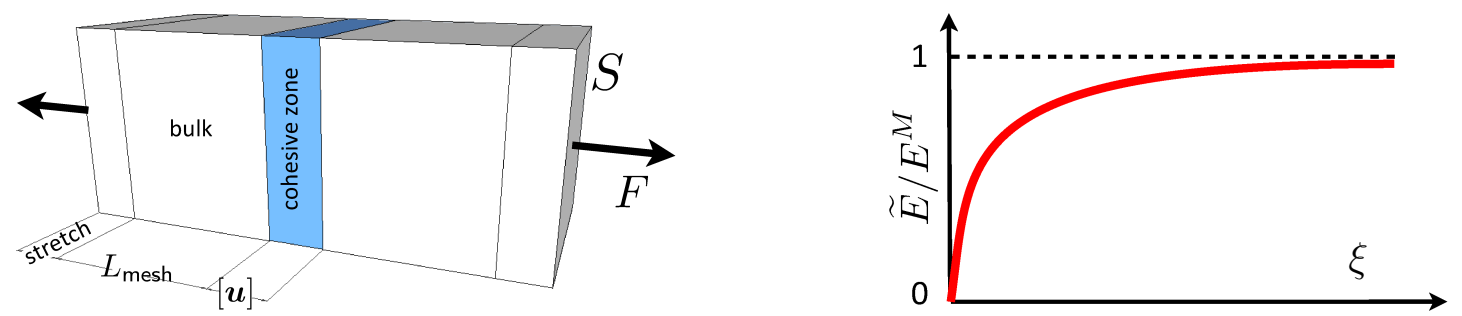

Fig. 1: Illustration of the relationship between apparent Young modulus and mesh size in 1D-case: (left) a representative part of 1D finite element mesh with embedded cohesive zone model, (right) overall Young modulus normalized by bulk modulus vs mesh size.

The aim of the present Note is to generalize these criteria to three dimensional situations and to any type of loadings. For that purpose, a new micromechanical model is developed.

\section{Micromechanical model}

\subsection{Cohesive-volumetric discretization as a matrix-inclusion composite}

Consider a cohesive-volumetric finite element discretization: each volumetric element is connected to each other using CZMs as boundary conditions. The central proposal is to replace this discretization with a continuous matrix containing cohesive inclusions (Fig. 2). The matrix has the same behavior as the
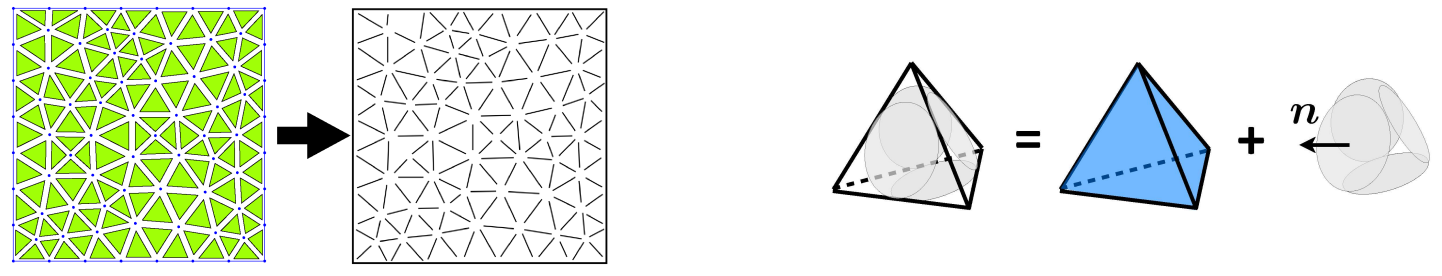

Fig. 2: Principle of the approach: a cohesive-volumetric finite element mesh is replaced by a continuous matrix corresponding to bulk elements and a collection of penny-shaped cohesive inclusions corresponding to the edges of the underlying mesh; (left) 2-D illustration, (right) 3-D illustration.

bulk finite element whereas the inclusions have a cohesive behavior defined by a traction-separation law. The spatial distribution of the cohesive inclusions corresponds to those of the edges of the underlying mesh and has the same density, denoted by $Z$. In particular, in the case of a statistically isotropic mesh, e.g. a Delaunay-type mesh, the inclusions are randomly distributed in space and in orientation. The density $Z$ corresponds to the specific 'surface' of the interface between the meshes: in two dimensions, $Z$ is equal to the ratio of the total length of the edges to the area of the meshed surface; in three dimensions, $Z$ is equal to the ratio of the total area of the edges to the volume of the meshed body. Moreover, the following geometric assumption is made:

H1: The inclusions are assumed to be of penny-shaped type (Fig. 2). In two dimensions, the straight edges of the mesh are thus replaced by zero thickness whiskers and this assumption has no consequence. In three dimensions, the polygonal edges are replaced by disks (with the same spatial density): the two situations are admittedly close to each other but are different when considered rigorously.

\subsection{Phases properties}

Subsequently, we restrict our attention to linear elastic behaviors for the matrix and for cohesive inclusions. The continuous matrix phase is considered as isotropic and its constitutive relation reads:

$$
\boldsymbol{\sigma}=\mathbb{C}^{M}: \boldsymbol{\varepsilon} \quad \text { with } \quad \mathbb{C}^{M}=3 k^{M} \mathbb{J}+2 \mu^{M} \mathbb{K},
$$


where $\sigma$ (resp. $\varepsilon$ ) is the stress (resp. strain) field, $\mathbb{C}^{M}$ is a fourth order elasticity stiffness tensor, $k^{M}$ and $\mu^{M}$ are the bulk modulus and the shear modulus respectively, $\mathbb{J}$ and $\mathbb{K}$ are the isotropic fourth order symmetric tensors ( $i$ being the second order identity tensor):

$$
\mathbb{J}=\frac{1}{3} \boldsymbol{i} \otimes \boldsymbol{i}, \quad \mathbb{K}=\mathbb{I}-\mathbb{J}, \quad \text { with } \quad \mathbb{I}_{i j k l}=\frac{1}{2}\left(\boldsymbol{i}_{i k} \boldsymbol{i}_{j l}+\boldsymbol{i}_{i l} \boldsymbol{i}_{j k}\right) .
$$

The cohesive behavior for the penny-shaped inclusions is defined by a traction-separation law which corresponds to a linear relation between the cohesive stress vector $\mathbf{R}^{\mathrm{coh}}$ and the opening displacement vector $[\boldsymbol{u}]$, in a local normal-tangent basis oriented by the normal vector $\boldsymbol{n}$ to the cohesive inclusion:

$$
\mathbf{R}^{\mathrm{coh}}=\boldsymbol{K} \cdot[\boldsymbol{u}] \quad \text { with } \quad \boldsymbol{K}=\left(C_{\mathrm{N}} \boldsymbol{n} \otimes \boldsymbol{n}+C_{\mathrm{\top}} \frac{\boldsymbol{u}_{\mathrm{\top}} \otimes \boldsymbol{u}_{\mathrm{\top}}}{\boldsymbol{u}_{\mathrm{\top}} \cdot \boldsymbol{u}_{\mathrm{\top}}}\right)
$$

where $C_{\mathrm{N}}$ (resp. $C_{\mathrm{T}}$ ) is the normal (resp. tangential) initial 'stiffness' of the cohesive law, $u_{\mathrm{N}}\left(\right.$ resp. $\boldsymbol{u}_{\mathrm{T}}$ ) is the normal (resp. tangential) component of $[\boldsymbol{u}]=u_{N} \boldsymbol{n}+\boldsymbol{u}_{\top}$. Hence, the cohesive stress tensor reads (1):

$$
\boldsymbol{\sigma}^{\mathrm{coh}}=\left(C_{\mathrm{N}} \mathbb{E}_{l}+C_{\mathrm{T}} \mathbb{K}_{l}\right):\left([\boldsymbol{u}] \otimes_{s} \boldsymbol{n}\right)
$$

where $\otimes_{s}$ is the symmetric dyadic product $\left(2 \boldsymbol{a} \otimes_{s} \boldsymbol{b}=a_{i} b_{j}+a_{j} b_{i}\right.$ for any vector $\boldsymbol{a}$ and $\left.\boldsymbol{b}\right)$ and $\mathbb{E}_{l}, \mathbb{K}_{l}$ are two components of the fourth order transversely isotropic and symmetric tensors frame: $\mathbb{E}_{l}=\boldsymbol{n} \otimes$ $\boldsymbol{n} \otimes \boldsymbol{n} \otimes \boldsymbol{n}$ and $\mathbb{K}_{l}=2\left(\boldsymbol{j}_{\boldsymbol{s}} \otimes \boldsymbol{j}_{\boldsymbol{s}}+\boldsymbol{j}_{\boldsymbol{t}} \otimes \boldsymbol{j}_{\boldsymbol{t}}\right)$ with $\boldsymbol{j}_{\boldsymbol{s}}=\boldsymbol{n} \otimes_{s} \boldsymbol{s}$ and $\boldsymbol{j}_{\boldsymbol{t}}=\boldsymbol{n} \otimes_{s} \boldsymbol{t}$, where $\boldsymbol{s}$ and $\boldsymbol{t}$ are two orthogonal vectors defining the transversal plane $(\boldsymbol{n}, \boldsymbol{t}, \boldsymbol{s}$ define the local orthogonal basis of the cohesive inclusion). A fourth order cohesive stiffness tensor $\mathbb{C}^{\text {coh }}$ is constructed, defining the cohesive 'strain' with the help of a small length $e(\underline{6})$, and the constitutive relation thus reads for the inclusions:

$$
\boldsymbol{\sigma}^{\text {coh }}=\mathbb{C}^{\text {coh }}: \varepsilon^{\text {coh }} \quad \text { with } \quad \mathbb{C}^{\text {coh }}=e\left(C_{\mathrm{N}} \mathbb{E}_{l}+C_{\mathrm{T}} \mathbb{K}_{l}\right) \quad \text { and } \quad \varepsilon^{\text {coh }}=\frac{[\boldsymbol{u}] \otimes_{s} \boldsymbol{n}}{e} .
$$

The small length $e$, that should tend to zero, is a fictitious thickness associated to the cohesive zone. Since oblate ellipsoids tend to penny-shaped inclusions when their thickness tends to zero, the cohesive inclusions are now considered as oblate ellipsoids and their volume fraction $f$ is the product of the density $Z$ (inversely proportional to a length, namely the mesh size $L_{\text {mesh }}$ ) and of the fictitious thickness $e: f=e Z$ (a cylindrical hypothesis instead of oblate one has no consequence when $e \rightarrow 0$ ).

\subsection{Hashin-Shtrikman estimate}

A Hashin-Shtrikman estimate (4) is used in order to establish an analytical expression of the overall elastic stiffness, denoted by $\mathbb{C}^{\text {hom }}$. The considered matrix-inclusion composite (Fig. 2) is composed of an isotropic elastic matrix (volume fraction $1-f$ ) following the constitutive relation (2) and of a collection of transversely isotropic oblate ellipsoids (volume fraction $f$ ) with the largest semi-axis $a$ varying from $A^{-}$to $A^{+}$, with the same aspect ratio $w \ll 1$ and following the constitutive relation (3). The orientation of these ellipsoids is characterized by the direction of their symmetry axis, i.e. a vector $\boldsymbol{n}$ of the unit sphere $S$ of $\mathbb{R}^{3}$ (measure $4 \pi$ ). Splitting this collection of inclusions into $N-1$ families $r(r=2, \ldots, N)$ and defining the inclusion density $\phi(a, \boldsymbol{n})$ such that $\phi(a, \boldsymbol{n}) d a d S$ is the number of inclusions of the $r$-th family with radius included in $(a, a+d a)$ and elementary surface $d S$ centred on $\boldsymbol{n}$. The volume fraction of the $r$-th family reads: $c_{r}=\phi(a, \boldsymbol{n}) \frac{4 \pi}{3} a^{3} w d a d s$, with $\sum_{r=2}^{N} c_{r}=f$. In this situation, the following generalization of the Hashin-Shtrikman's result holds (14):

$$
\mathbb{C}^{\mathrm{HS}}\left(\mathbb{C}^{0}\right)=\left[(1-f) \mathbb{C}^{M}: \mathbb{V}_{1}+\sum_{r=2}^{N} c_{r} \mathbb{C}_{r}^{\mathrm{coh}}: \mathbb{V}_{r}\right]:\left[(1-f) \mathbb{V}_{1}+\sum_{r=2}^{N} c_{r} \mathbb{V}_{r}\right]^{-1}
$$

where $\mathbb{C}^{0}$ (resp. $\mathbb{C}_{r}^{\text {coh }}$ ) is the fourth order stiffness tensor of a reference medium (resp. of the $r$-th family of inclusions), $\mathbb{V}_{1}=\left(\mathbb{C}^{*}+\mathbb{C}^{M}\right)^{-1}$ and $\mathbb{V}_{r}=\left(\mathbb{C}^{*}+\mathbb{C}_{r}^{\text {coh }}\right)^{-1}$. The Hill influence tensor 
$\mathbb{C}^{*}$ characterizes the stress that the infinite surrounding reference medium applies on a single ellipsoid having a homogeneous eigenstrain: $\mathbb{C}^{*}$ depends on the shape of the inclusion $w$, on its orientation $\boldsymbol{n}$ and on the reference medium $\mathbb{C}^{0}$. Introducing $\mathbb{T}_{r}=\mathbb{V}_{r}: \mathbb{V}_{1}^{-1}$ and remarking that cohesive stiffness depends only on the orientation $\boldsymbol{n}$, i.e. $\mathbb{C}_{r}^{\mathrm{coh}}=\mathbb{C}^{\mathrm{coh}}(\boldsymbol{n})$, and that tensor $\mathbb{T}_{r}$ depends only on the aspect ratio $w$ and the direction $n$, i.e.

$$
\mathbb{T}_{r}=\mathbb{T}(w, \boldsymbol{n})=\left(\mathbb{C}^{*}(w, \boldsymbol{n})+\mathbb{C}^{\operatorname{coh}}(\boldsymbol{n})\right)^{-1}:\left(\mathbb{C}^{*}(w, \boldsymbol{n})+\mathbb{C}^{M}\right)
$$

the estimate (6) is rewritten with the help of the density $\phi(a, \boldsymbol{n})$ and an integration over the radius and the orientation:

$$
\begin{aligned}
\mathbb{C}^{\mathrm{HS}}\left(\mathbb{C}^{0}\right)= & {\left[(1-f) \mathbb{C}^{M}+\int_{S} \int_{A^{-}}^{A^{+}} \phi(a, \boldsymbol{n}) \frac{4 \pi}{3} a^{3} w \mathbb{C}^{\mathrm{coh}}(\boldsymbol{n}): \mathbb{T}(w, \boldsymbol{n}) d a d S\right]: } \\
& {\left[(1-f) \mathbb{I}+\int_{S} \int_{A^{-}}^{A^{+}} \phi(a, \boldsymbol{n}) \frac{4 \pi}{3} a^{3} w \mathbb{T}(w, \boldsymbol{n}) d a d S\right]^{-1} . }
\end{aligned}
$$

Since $w$ is constant and $\mathbb{C}^{\mathrm{coh}}(\boldsymbol{n})$ and $\mathbb{T}(w, \boldsymbol{n})$ do not depend on radius $a$, integrating over $a$ leads to:

$$
\mathbb{C}^{\mathrm{HS}}\left(\mathbb{C}^{0}\right)=\left[(1-f) \mathbb{C}^{M}+w \int_{S} \psi(\boldsymbol{n}) \mathbb{C}^{\mathrm{coh}}(\boldsymbol{n}): \mathbb{T}(w, \boldsymbol{n}) d S\right]:\left[(1-f) \mathbb{I}+w \int_{S} \psi(\boldsymbol{n}) \mathbb{T}(w, \boldsymbol{n}) d S\right]^{-1}
$$

where $\psi(\boldsymbol{n})=\int_{A^{-}}^{A^{+}}(4 / 3) \pi a^{3} \phi(a, \boldsymbol{n}) d a$. If the underlying mesh contains specific orientations (e.g. anisotropic meshes), they have to be taken into account through the density $\psi(\boldsymbol{n})$. For simplicity, isotropic meshes (e.g. Delaunay-type meshes) alone are considered in the next section.

\subsection{Lower bound for isotropic meshes}

Assuming that the orientations of inclusions have an equi-probability property, the density $\psi(\boldsymbol{n})$ reduces to $f /(4 \pi w)$ and the estimate (9) becomes with $f=e Z$ :

$$
\begin{aligned}
\mathbb{C}^{\mathrm{HS}}\left(\mathbb{C}^{0}\right) & =\left[(1-f) \mathbb{C}^{M}+f \frac{1}{4 \pi} \int_{S} \mathbb{C}^{\mathrm{coh}}(\boldsymbol{n}): \mathbb{T}(w, \boldsymbol{n}) d S\right]:\left[(1-f) \mathbb{I}+f \frac{1}{4 \pi} \int_{S} \mathbb{T}(w, \boldsymbol{n}) d S\right]^{-1} \\
& =\left[(1-e Z) \mathbb{C}^{M}+e Z\left\langle\mathbb{C}^{\mathrm{coh}}: \mathbb{T}\right\rangle_{\circlearrowleft}\right]:\left[(1-e Z) \mathbb{I}+e Z\langle\mathbb{T}\rangle_{\circlearrowleft}\right]^{-1}
\end{aligned}
$$

where, for any fourth order tensor $\mathbb{D},\langle\mathbb{D}\rangle_{\circlearrowleft}=(\mathbb{J}:: \mathbb{D}) \mathbb{J}+(1 / 5)(\mathbb{K}:: \mathbb{D}) \mathbb{K}$ indicates the average on all orientations (3). The overall stiffness tensor is obtained as $\mathbb{C}^{\text {hom }}=\lim _{e \rightarrow 0} \mathbb{C}^{\mathrm{HS}}\left(\mathbb{C}^{0}\right)$.

At this stage, according to the choice of the reference medium $\mathbb{C}^{0}$, different bounds and estimates can be derived. Since, in a quadratic sense, $\mathbb{C}^{\text {coh }}$ is smaller than the matrix elastic tensor $\mathbb{C}^{M}$ when $e$ tends to zero (see equation (5)), the Hashin-Shtrikman upper bound or the Mori-Tanaka estimate correspond to the choice $\mathbb{C}^{0}=\mathbb{C}^{M}$ which gives the trivial result $\mathbb{C}^{\text {hom }}=\mathbb{C}^{M}$ at the limit $e \rightarrow 0$. The same trivial result is obtained for the self-consistent scheme $\left(\mathbb{C}^{0}=\mathbb{C}^{\text {hom }}\right)$. Thus, by focusing on the case $\mathbb{C}^{0}=\mathbb{C}^{\text {coh }}$, the Hashin-Shtrikman lower bound is defined:

$$
\mathbb{C}^{\text {hom }}=\lim _{e \rightarrow 0} \mathbb{C}^{\mathrm{HS}}\left(\mathbb{C}^{\mathrm{coh}}\right)=\lim _{e \rightarrow 0}\left[(1-e Z) \mathbb{C}^{M}+e Z\left\langle\mathbb{C}^{\mathrm{coh}}: \mathbb{T}\right\rangle_{\circlearrowleft}\right]:\left[(1-e Z) \mathbb{I}+e Z\langle\mathbb{T}\rangle_{\circlearrowleft}\right]^{-1} .
$$

Using the method proposed in (9) to estimate the transversely isotropic influence tensor $\mathbb{C}^{*}\left(\mathbb{C}^{\text {coh }}\right)$ and therefore the tensor $\mathbb{T}$ in (11), see (7), the expression of the bulk and shear effective moduli is derived after a convenient passage to the limit for $e \rightarrow 0$ :

$$
\frac{k^{\text {hom }}}{k^{M}}=\frac{\xi_{\mathrm{k}}}{\xi_{\mathrm{k}}+1} \quad \text { with } \quad \xi_{\mathrm{k}}=\frac{C_{\mathrm{N}}}{Z k^{M}} \quad \text { and } \quad \frac{\mu^{\text {hom }}}{\mu^{M}}=\frac{\xi_{\mu}}{\xi_{\mu}+1} \quad \text { with } \quad \xi_{\mu}=\frac{15}{4\left(1+3 C_{\mathrm{N}} / C_{\mathrm{T}}\right)} \frac{C_{\mathrm{N}}}{Z \mu^{M}} \text {. }
$$


It is worth noting that equations (12) show that a cohesive-volumetric formulation with vanishing tangential cohesive stiffness $\left(C_{\mathrm{T}} \rightarrow 0\right)$ leads to a macroscopic no shear material ( $\left.\mu^{\text {hom }} \rightarrow 0\right)$. Moreover, the lower bounds (12) allow the definition of criteria on the overall loss of stiffnesses, e.g. assuming that a reduction of $5 \%$ on the apparent bulk modulus and shear modulus is admissible:

$$
\frac{k^{\text {hom }}}{k^{M}} \geq 0.95 \text { is ensured for } \xi_{\mathrm{k}} \geq 20 \text { and } \frac{\mu^{\text {hom }}}{\mu^{M}} \geq 0.95 \text { is ensured for } \xi_{\mu} \geq 20 .
$$

In the next section, the lower bounds (12) and the (13)-type criteria are numerically tested in the 2-D case for isotropic and regular meshes. As mentioned in assumption H1, no approximation is introduced by the penny-shaped morphology in that case and (12) is a rigorous lower bound for the cohesive-volumetric approach with isotropic meshes.

\section{Numerical comparisons}

The numerical experiments concern both 2-D Delaunay and regular 'cross-triangle quadrilateral' meshes (each square element is subdivided into four isosceles triangles). The linear elastic response of cohesivevolumetric finite element method is calculated under plane-strain conditions (software XPER $(7 ; 8)$ ). The comparison between numerical results and relations (12) involves the computation of the density $Z=\mathcal{A} / \mathcal{S}$ where $\mathcal{A}$ is the total edge length (resp. area) in 2-D (resp. in 3-D) and $\mathcal{S}$ is the total area (resp. volume) of the 2-D (resp. 3-D) meshed body. For regular meshes characterized by a mesh size $L_{\text {mesh }}, \mathcal{A}$ is proportional to $L_{\text {mesh }}^{q-1}$ and $\mathcal{S}$ is proportional to $L_{\text {mesh }}^{q}$ where $q$ is the considered dimension; density $Z$ is thus inversely proportional to the mesh size $Z=\gamma / L_{\text {mesh }}$ where $\gamma$ depends on the spatial distribution of the underlying mesh. For cross-triangle quadrilateral 2-D meshes, $\gamma=2(1+\sqrt{2})(1 ;$; 13). For 2-D or 3 -D Delaunay tessellations, $L_{\text {mesh }}$ is a stochastic parameter and $\gamma$ has to be understood as a mean value and a variance $(11)$.
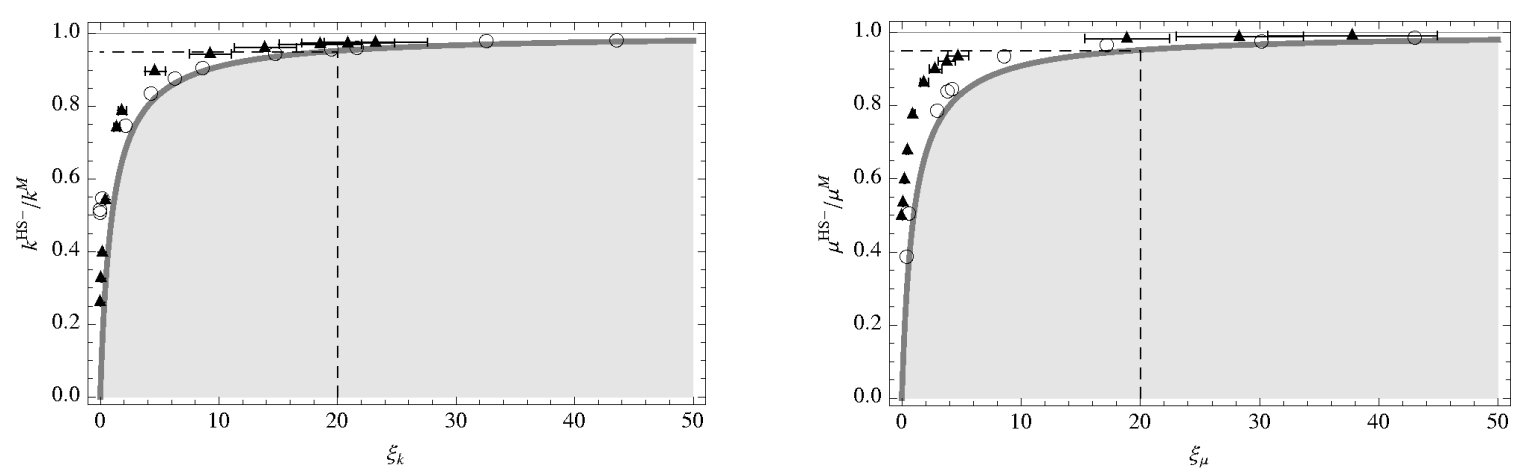

Fig. 3: Normalized overall elastic modulus of a cohesive-volumetric formulation with intrinsic CZMs (case $C_{\mathrm{N}}=C_{\mathrm{T}}$ ): lower bounds (12) (thick gray line), criteria (13) (dashed line), numerical results for Delaunay meshes (closed symbols with variance) and for cross-triangle quadrilateral meshes (open symbols); bulk modulus (left) and shear modulus (right).

Typical results for the particular case $C_{\mathrm{N}}=C_{\mathrm{T}}$ are shown in Fig. 3. As expected, relations (12) are rigorous lower bounds for isotropic meshes (Delaunay) and the numerical results clearly show that criteria (13), $\xi_{\mathrm{k}} \geq 20$ and $\xi_{\mu} \geq 20$, ensure that the overall added compliance do not exceed $5 \%$ (dashed lines in Fig. 3). Moreover, these results illustrate that (12) are convenient estimates for regular meshes. Noting that these regular meshes exhibit a higher added compliance than isotropic meshes, we derive in the next section criteria on cohesive stiffnesses. 


\section{Criteria on cohesive stiffnesses}

Following (1), criteria on cohesive stiffnesses can be obtained from the previous micromechanical model. Considering a homogeneous medium with a Young modulus $E^{\text {hom }}=9 k^{\text {hom }} \mu^{\text {hom }} /\left(3 k^{\text {hom }}+\right.$ $\left.\mu^{\text {hom }}\right)$ and a Poisson ratio $\nu^{\text {hom }}=\left(3 k^{\text {hom }}-2 \mu^{\text {hom }}\right) /\left(6 k^{\text {hom }}+2 \mu^{\text {hom }}\right)-$ the same relations hold for the matrix phase - two relative apparent reductions $R$ (resp. $r$ ) due to the presence of intrinsic CZMs are defined for Young modulus (resp. Poisson ratio) with the help of relations (12):

$$
\begin{gathered}
R=\frac{E^{\text {hom }}}{E^{M}}=\frac{\xi_{\mathrm{E}}}{1+\xi_{\mathrm{E}}} \quad \text { where } \quad \xi_{\mathrm{E}}=\frac{5}{1+(4 / 3)\left(C_{\mathrm{N}} / C_{\mathrm{T}}\right)} \times \frac{C_{\mathrm{N}}}{E^{M} Z} \\
r=\frac{\nu^{\text {hom }}}{\nu^{M}}=\frac{E^{M} Z\left(-1+2 C_{\mathrm{N}} / C_{\mathrm{T}}\right)+15 C_{\mathrm{N}} \nu^{M}}{E^{M} Z\left(3+4 C_{\mathrm{N}} / C_{\mathrm{T}}\right) \nu^{M}+15 C_{\mathrm{N}} \nu^{M}} .
\end{gathered}
$$

The relation (14) extends the 1D-equation (1) with a prefactor on $\xi$ and the use of the density $Z$ instead of $L_{\text {mesh }}$. Reduction $R=1$ can not be reached (the overall Young modulus is always disturbed by the presence of CZMs), but for a given value of $R \in[0,1$ [, equation (14) leads to an estimate by lower value of $C_{\mathrm{N}} /\left(E^{M} Z\right)$ involving the ratio $C_{\mathrm{N}} / C_{\mathrm{T}}$ :

$$
\frac{C_{\mathrm{N}}}{E^{M} Z} \geq \frac{1}{5} \frac{R}{1-R}\left(1+\frac{4}{3} \frac{C_{\mathrm{N}}}{C_{\mathrm{T}}}\right)
$$

Fortunately, apparent reduction $r=1$, i.e. $\nu^{\text {hom }}=\nu^{M}$, can be reached in (15) fixing the ratio $C_{\mathrm{N}} / C_{\mathrm{T}}$ and thus defining 'invisible' intrinsic CZMs for the Poisson ratio of the considered homogeneous medium:

$$
\nu^{\text {hom }}=\nu^{M} \Rightarrow \frac{C_{\mathrm{N}}}{C_{\mathrm{T}}}=\frac{1}{2} \frac{1+3 \nu^{M}}{1-2 \nu^{M}} .
$$

The condition (17) ensures same elastic reduction for bulk, shear and Young moduli: $k^{\text {hom }} / k^{M}=$ $\mu^{\text {hom }} / \mu^{M}=E^{\text {hom }} / E^{M}$; same relations were proposed by (5) as a closure condition in their selfconsistent estimate for pure hydrostatic loadings. The present approach can thus be seen as an improvement of the results of (5) to arbitrary type of loadings. Recalling that $Z=\gamma / L_{\text {mesh }}$, one obtains with (16) and (17) a criterion on the ratio $C_{\mathrm{N}} L_{\text {mesh }} / E^{M}$ extending semi-empirical criteria previously proposed in the literature $(2 ; 13)$ :

$$
\frac{C_{\mathrm{N}} L_{\mathrm{mesh}}}{E^{M}} \geq \gamma \frac{R}{1-R} \frac{1}{3\left(1-2 \nu^{M}\right)} .
$$

As shown in section 3, the case of regular meshes with $\gamma=2(1+\sqrt{2})$ allows the derivation of 2-D practical criteria on the cohesive stiffnesses. This value of $\gamma$ combined with the relation (17) and the inequality (18) provides 'practical $5 \%$ criteria' $(R=0.95)$ :

$$
\frac{C_{\mathrm{T}}}{C_{\mathrm{N}}}=2 \frac{1-2 \nu^{M}}{1+3 \nu^{M}} \quad \text { with } \quad \frac{C_{\mathrm{N}} L_{\text {mesh }}}{E^{M}} \geq \frac{30}{1-2 \nu^{M}} .
$$

For a bulk material with $\nu^{M}=0.2$, these criteria give $C_{\mathrm{T}}=0.75 C_{\mathrm{N}}$ and $C_{\mathrm{N}} \geq 50 E^{M} / L_{\text {mesh }}$. Conversely, for a bulk material with $\nu^{M}=0.2$, the criterion proposed by (2), i.e. $C_{\mathrm{N}} L_{\text {mesh }} / E^{M} \geq 10$, corresponds to a reduction $R$ about 0.78 , which guaranties only that the loss in apparent Young modulus is less than $22 \%$.

\section{Conclusion}

In this Note, we have studied the overall linear constitutive behavior of an isotropic medium containing intrinsic cohesive inclusions considered as a representation of a cohesive-volumetric finite element 
modeling. An asymptotic formulation of the Hashin-Shtrikman lower bound with convenient orientational average allows the definition - for any type of loading - rigorous criteria (12) on the normal $\left(C_{\mathrm{N}}\right)$ and tangential $\left(C_{\mathrm{T}}\right)$ cohesive stiffnesses for isotropic meshes and practical criteria for regular meshes. The accuracy of the proposed criteria were tested by numerical comparisons. Intermediate results of this study show that: $1 /$ a cohesive-volumetric approach with a vanishing tangential cohesive stiffness $\left(C_{\mathrm{T}} \rightarrow 0\right)$ leads to an overall no shear material, 2/ an a priori estimate (19) allows the calibration of $C_{\mathrm{N}}$ and $C_{\mathrm{T}}$ as a function both of the mesh size and of the elastic properties of the bulk material. The extension of this results to damageable cohesive laws will be presented in a forthcoming work.

\section{Acknowledgements}

The authors are indebted to Dr. M. Bornert for useful suggestions in orientational averaging and to Dr. F. Perales for providing XPER software facilities.

\section{References}

[1] V. Acary, Y. Monerie, Non-smooth fracture dynamics using a cohesive zone approach, Tech. Rep. RR-6032, Institut National de Recherche en Informatique et en Automatique (2006).

[2] H. Espinosa, P. Zavattieri, A grain level model for the study of failure initiation and evolution in polycrystalline brittle materials. Part I: Theory and numerical implementation, Mechanics of Materials 35 (2003) 333 - 364 .

[3] J.-M. Gatt, Y. Monerie, D. Laux, D. Baron, Elastic behavior of porous ceramics: application to nuclear fuel materials, Journal of Nuclear Materials 336 (2005) 145 - 155.

[4] Z. Hashin, S. Shtrikman, A variational approach to the theory of the elastic behaviour of multiphase materials, Journal of the Mechanics and Physics of Solids 11 (1963) 127-140.

[5] S. Li, G. Wang, On damage theory of a cohesive medium, International Journal of Engineering Science 42 (2004) 861-885.

[6] J.-C. Michel, P. Suquet, F. Thébaud, Une modélisation du rôle des interfaces dans le comportement des composites à matrice métallique, Revue Européenne des Eléments Finis 4 (1994) 573-595.

[7] F. Perales, S. Bourgeois, A. Chrysochoos, Y. Monerie, Two field multibody method for periodic homogenization in fracture mechanics of nonlinear heterogeneous materials, Engineering Fracture Mechanics 75 (2008) 3378-3398.

[8] F. Perales, F. Dubois, Y. Monerie, B. Piar, L. Stainier, Multi-body NSCD strategy as a multi-domain solver. Application to code coupling dedicated to the modeling of fracture of heterogeneous media, European Journal of Computational Mechanics, 19 (2010) 389-417.

[9] I. Sevostianov, N. Yilmaz, V. Kushch, V. Levin, Effective elastic properties of matrix composites with transversely-isotropic phases, International journal of solids and structures 42 (2005) 455476.

[10] S. Song, G. Paulino, W. Buttlar, A bilinear cohesive zone model tailored for fracture of asphalt concrete considering viscoelastic bulk material, Engineering Fracture mechanics 73 (2006) 2829_ 2848 .

[11] D. Stoyan, W. Kendall, J. Mecke, Stochastic geometry and its applications, John Wiley and Sons, Chichester, New York, Brisbane, Toronto, Singapore, 1995. 
[12] M. Tijssens, L. Sluys, E. van der Giessen, Numerical simulation of quasi-brittle fracture using damaging cohesive surfaces, European Journal of Mechanics A/Solids 19 (2000) 761-779.

[13] V. Tomar, J. Zhai, M. Zhou, Bounds for element size in a variable stiffness cohesive finite element model, International Journal for Numerical Methods in Engineering 61 (2004) 1894-1920.

[14] J.R. Willis, Bounds and self-consistent estimates for the overall properties of anisotropic composites, IJournal of the Mechanics and Physics of Solids 25 (1977) 185-202. 